\title{
PSD signal conditioning device
}

\begin{abstract}
Zhen Yang
School of North China Electric Power University Baoding, Baoding 071000, China; 649066081@qq.com

Abstract: A PSD signal acquisition and processing system is designed based on the new single-chip stm32 and the segmented psd, and the data display interface circuit is designed by using the liquid crystal display module for the PSD signal output characteristic and the portable application in engineering practice. The real-time acquisition and display of the PSD output signal are carried out. The experiment shows that the acquisition system is feasible and meets the design requirements.
\end{abstract}

Keywords: PSD, Signal processing, Circuit design.

\section{Introduction}

Position Sensitive Sensor PSD (Position Sensitive Device) is a kind of sensor that can track the position of the beam irradiated to its surface. Its basic structure includes high resistance substrate and one or two layers of resistive layer covering the surface of the substrate For one-dimensional or two-dimensional PSD).

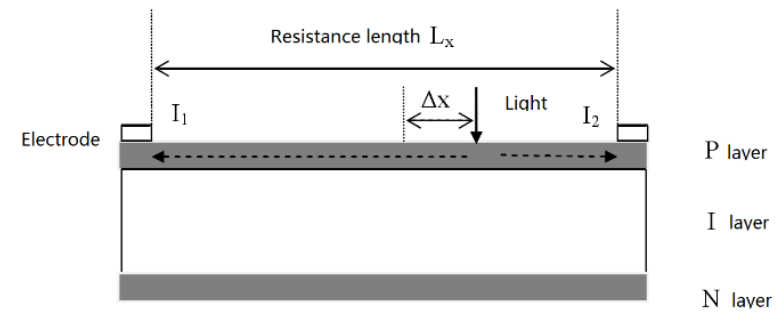

Figure 1 one-dimensional PSD schematic

PSD sensing mechanism is very simple: If the top of the P layer is a beam of light, there will be a corresponding charge generation, the charge size is proportional to the intensity of light, the resistance layer formed in the potential will lead to PSD surface spot and resistance The flow of photocurrent between the two electrodes at both ends of the layer.

\section{Hardware system design}

\section{1 overall signal processing circuit}

According to the operation mode of PSD, it can be seen that the electrical signal obtained from the PSD electrode can not be directly expressed in position. A commonly used PSD signal processing method is to combine the amplification circuit, analog computing circuit, analog-digital conversion circuit, the first signal amplification, and then the electrical signal addition, subtraction and division of the arithmetic processing, the spot position and voltage values Relationship, and then the 
analog position signal into a digital signal, easy to computer processing.

Therefore, the hardware block diagram of the signal processor used in this paper is shown in Figure 2 below:

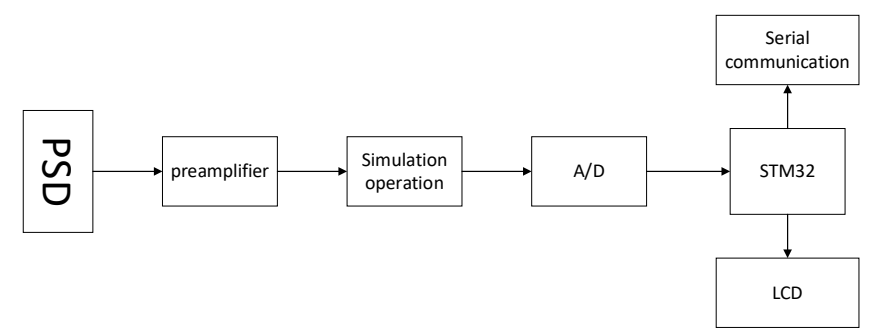

Fig 2 Hardware block diagram

\subsection{PSD choice}

In this paper, we chose the location resolution higher segment PSD, it has a very good stability, fast response, The segmented PSD typically divides the base semiconductor into two parts by a spacer that does not produce a photoelectric effect or Four parts,As shown in Figure 3, a schematic diagram of the signal is generated for the PSD element.

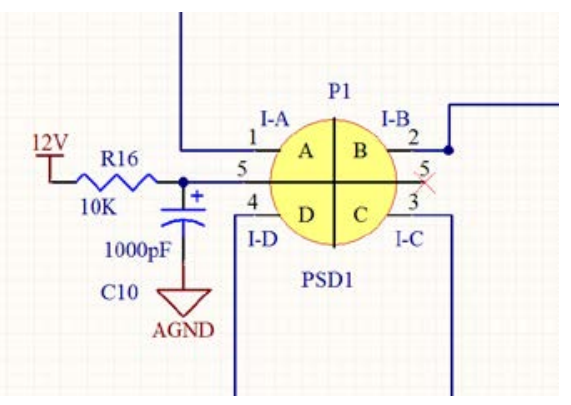

Figure 3 Schematic diagram of PSD components

The beam is irradiated to the surface of the PSD, the PSD element generates the current signal I-A, I-B, I-C,I-D, and the current of the electrode is calculated to obtain the relative position of the beam. If the spot is located at the center of the PSD, the four electrodes of the PSD can theoretically produce the same photocurrent, The relative position of the beam can be expressed as:

$$
\begin{aligned}
& X=\frac{\left(I_{B}+I_{C}\right)-\left(I_{A}+I_{D}\right)}{I_{A}+I_{B}+I_{C}+I_{D}} \\
& Y=\frac{\left(I_{A}+I_{B}\right)-\left(I_{C}+I_{D}\right)}{I_{A}+I_{B}+I_{C}+I_{D}}
\end{aligned}
$$

Where $\mathrm{X}$ and $\mathrm{Y}$ are the relative positions of the beam in the $\mathrm{X}$-axis and $\mathrm{Y}$-axis directions of the PSD, IA, IB, IC, and ID are the output currents of the four electrodes of the PSD.

\section{3 preamplifier circuit}

In the position measurement, the PSD of the electrodes will produce $0-100 \mathrm{uA}$ weak current, these current signals need to be converted into a voltage value can be carried out in the signal data processing operations, while the current signal is too small, so 
the need for the current signal to do Magnify, and then perform mathematical operations.

Through the current-voltage conversion circuit, the input current I can be converted to the input voltage $\mathrm{V}_{0}$,

$$
\mathrm{V}_{0}=-\mathrm{I} * \mathrm{R}_{\mathrm{F}}
$$

Which is the feedback resistor in the circuit. In addition, the amplifier circuit magnification $\mathrm{A}_{\mathrm{OUT}}$, by the resistance of the resistance of the connection with the base of the resistance value of the ratio of the decision, through the conversion operation, you can get the voltage value:

$V_{\text {ouT }}=I * R_{F} * \frac{R_{F}}{R_{0}}$

The formula shows that the output voltage $\mathrm{V}_{\text {OuT }}$ is proportional to the input current and the scale factor is determined by the resistance values in the switching circuit. Therefore, different resistors are selected based on the appropriate current magnification. The magnification ratio chosen here is 1: 0000. Get a maximum voltage of $5 \mathrm{~V}$.

The PSD signal amplification circuit shown in Figure 4, the op amp uses OPA37FZ amplifier, the amplifier has a small offset, responsive, high precision, low noise and other advantages, you can get more accurate measurement data. Capacitance is used for high frequency noise reduction。Figure 4 PSD signal amplification circuit diagram

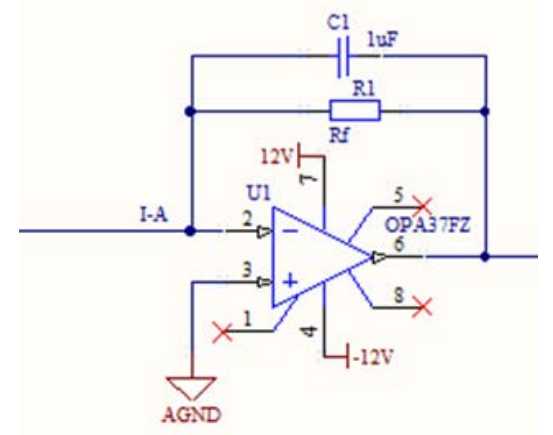

Fig4 signal amplification circuit diagram

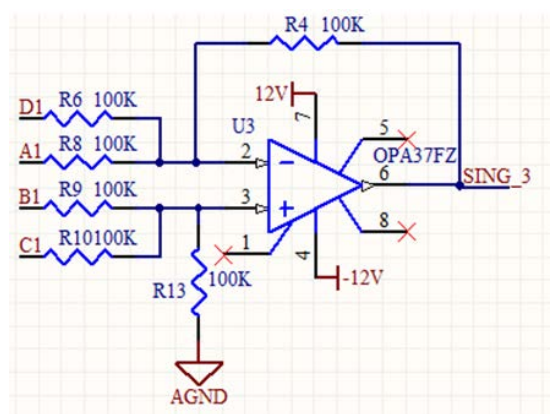

Fig5 Signal addition and subtraction

\section{4 analog operation circuit}

As can be seen from the above, to get the position coordinates of the spot on the PSD element, it is necessary to perform the mathematical operation on the output signal of the PSD. These operations are done by means of the analog operation circuit. Therefore, the mathematical operation part of the analog operation circuit is shown in Fig. 5The two signals of the spot coordinates (X, Y) can be expressed as

$$
\begin{aligned}
& X=\frac{(A+D)-(B+C)}{A+B+C+D} \\
& Y=\frac{(A+B)-(C+D)}{A+B+C+D}
\end{aligned}
$$


The above-mentioned two parts of the molecular part of the two amplifiers by the addition and subtraction operations, and the two denominator of the same, are $(\mathrm{A}+\mathrm{B}$ $+\mathrm{C}+\mathrm{D}$ ), by another amplifier to be in phase addition.

The arithmetic operation part of the analog operation circuit is passed to the two dividers after the operation of the amplifiers, and the AD534TD simulation chip is selected by the division operator. The chip has good linear performance and low input and output offset voltage And other advantages, you can achieve the system low noise, accurate requirements.

\subsection{STM32 data processing and TFTLED display module}

Output A, B, C, D 4 signal, need to occupy the A / D chip 4 channels, the chip work in single-ended mode to meet the four kinds of signal A / D sample value of the order of reading. STM32 calculated coordinates of the value through the LCD display, the use of LCD monitors and controllers have been together with the display module, and the use of single-chip parallel or serial interface to control the liquid crystal display module is being widely used. The method is simple and flexible, does not need to produce too many hardware circuits and very short development cycle characteristics, is a high efficiency, low cost control system information output solution

\section{3 .Results}

Using the P S D signal conditioning circuit described herein, a segment P S D (effective photosensitive surface $13 \mathrm{~m} \mathrm{~m} \times 13 \mathrm{~m} \mathrm{~m}$ )Line test, before the test of the laser spot drift, the accuracy of the micro-platform, such as the detection of scanning measurement step $1000 \mu \mathrm{m}$, spot diameter of about $200 \mu \mathrm{m}$, measured non-linear correction of the location shown in Figure 6 , The position of the non-linear correction is shown in Fig

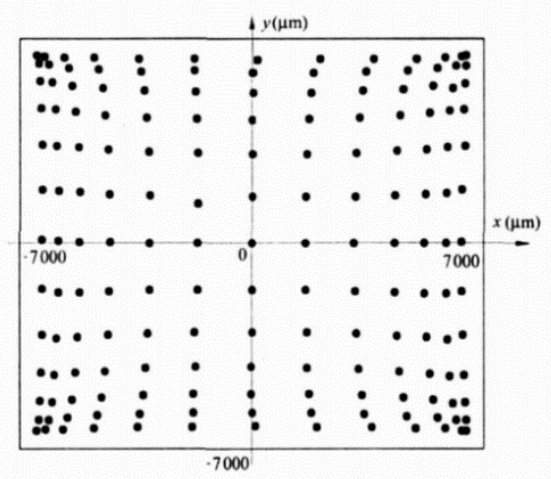

Fig6 Non- signal conditioning

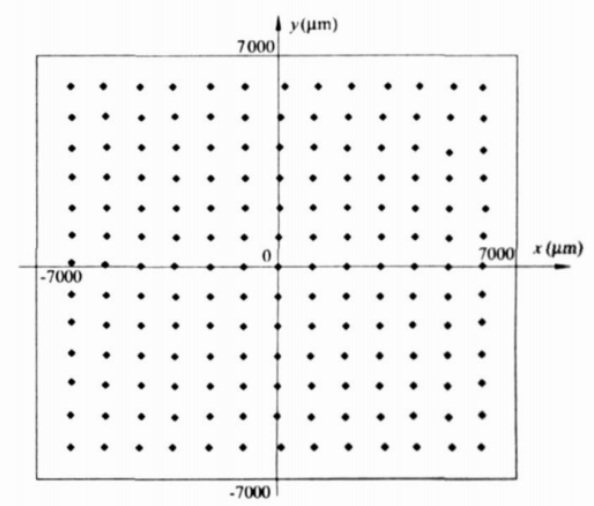

Fig7 signal conditioning

Figure 6, Figure 7 shows that: non-processed correction of the location of non-linear serious, not only the edge position error,Even the middle of the deviation is also great, which is provided with the results of the manufacturers are consistent with the signal conditioning position is very good.

\section{Reference}

[1] Feimeng Zhang,Yi Li,Hao Li, et al.Compensation method of PSD's 
backgroundlight based on improved BP neural network. IMSNA . 2012

[2] G. Lucovskey.Photoeffects in non-uniformly irradiated P-N junctions. Journal of Applied Physics . 1960

[3] HAFEZ M,SIDLER T C,SALATHE R P,et al.Design,simulation and experimental investigation of a compact single mirror tip/tilt laser scanner. Mechatronics . 2000 